\title{
Evaluation of Antioxidant Activities of Peptides Isolated from Korean Fermented Soybean Paste, Chungkukjang
}

\author{
Sun-Lim Kim*', Hee-Youn Chi****, Jung-Tae Kim*, On-Sook Hur**, Deog-Su Kim*, Sae-Jung Suh***, \\ Hyun-Bok Kim**, and III-Min Cheong***** \\ *National Institute of Crop Science RDA Suwon 441-857, Korea \\ **National Academy of Agricultural Science, RDA, Suwon, 441-707, Korea \\ ***International Technology Cooperation Center, RDA, Suwon, 441-707, Korea \\ ****MWorks, Co., LTD. Geumcheon-Ku, Seoul, 153-769, Korea \\ *****Department of Applied Life Science, College of Life and Environment Science, Konkuk University, \\ Seoul 143-701, Korea
}

\begin{abstract}
The objectives of present study were to characterize the peptides which were isolated from Korean fermented soybean paste, chungkukjang, and to determine their antioxidant activities. Four fractions were collected from the methanol extract of chungkukjang by using a recycling preparative HPLC. Among fractions, Fr-2 was identified to be highly potent free radical scavenging activity in the assay of 1,1-diphenyl-2-picryl-hydrazyl (DPPH) and nitroblue tetrazolium(NBT)-reduction inhibition. Base on antioxidant effects, fraction Fr-2 was employed for the refraction with a prep-column and separated into five fractions of which two fractions were identified to have higher antioxidant activity. To confirm the amino acid constituents of antioxidant fractions Fr-2-2 and Fr-2-3 were analyzed, and eight kinds of amino acids such as aspartic acid, threonine, serine, glutamic acid, glycine, lysine, histidine, and arginine were identified as the constituent amino acids. Antioxidant activities of the separated peptides were further assessed cell viability with 3-(4,5-dimethylthiazol-2-yl)-2,5-diphenyl terazolium bromide (MTT), and fluorescence-activated cell sorting (FACS) analysis of H4IIE cells treated with hydrogen peroxide $\left(\mathrm{H}_{2} \mathrm{O}_{2}\right)$. Chungkukjang peptides have shown their ability to protect $\mathrm{H} 4 \mathrm{IIE}$ rat hepatoma cells against $\mathrm{H}_{2} \mathrm{O}_{2-}$ induced oxidative stress by concentration and time-dependent manner. Therefore, These results indicated that fermented soybean paste chungkukjang will be promoted the antioxidant and radical scavenging activities, and beneficial for health. The antioxidant peptide fractions Fr-2-2 and Fr-2-3 were denominated as P-NICS-1 and P-NICS-2, respectively. However, further studies were required to clarify their amino acid sequences and molecular properties, and physiological significances.
\end{abstract}

Keywords : chungkukjang, antioxidant, peptide, amino acid, DPPH, NBT-reduction, cell viability, DCFH-DA oxidation

Soybean (Glycine $\max$ L.) contains approximately 40 percent protein, and this produces various peptide and amino acid during processes like fermentation.

Fermented soy foods such as chungkukjang and doenjang are important source of the Korean diet. Fermentation period of chungkukjang is relatively shorter than other traditional fermented soybean pastes.

Korean chungkukjang and Japanese natto are very similar products, but the differences between chungkukjang and natto are mainly found in the different soybean-seed size, fermentation process, and consumption method (Choe et al., 1996).

Chungkukjang is produced by fermentation with a cooked soybean, but their quality are varies considerably with soybean varieties, fermenting bacterial strains, fermentation time, sub-ingredients, and so on (Lee et al., 2005).

During the fermentation, chungkukjang produces considerable amounts of ferments by Bacillus substilis. The ferments decompose carbohydrates and protein, and simultaneously produce a sticky substance composed of fructan in the form of polyglutamate (Fujii et al., 1975). Polyglutamates produced in sufficiently fermented chungkukjang are sticky mucilage like spun thread and have a soft texture and

\footnotetext{
${ }^{\dagger}$ Corresponding author: (Phone) +82-31-290-6746 (E-mail) kimsl@korea.kr

$<$ Received 19 September 2011; Revised 14 November 2011; Accepted 19 November 2011>
} 
characteristic flavor and taste (Lee et al., 1991; Thomas et al., 2005).

The consumption of chungkukjang has been increasing rapidly in recent years due to its health beneficial properties. Several studies have demonstrated the biological activities of chungkukjang such as anticancer, antihypertensive activity, hypocholesterolemic and hypolipidemic effects (Chung et al., 1997; Kim et al., 1996; Lee et al., 1996; Kang et al., 2003).

Generally, peptide is known not only as a nutrient but for functions in numerous biological reactions (Mahmoud, 1994). Especially, various peptides derived from fermented soybean foods are known as the most potent physiological agents (Lee, et al., 1998).

Oligopeptide is composed from bonding of 2 to 10 amino acids, and it means below 10,000 of normal molecular weight. Peptide existing inside human bodies shows many physiological activities, therefore interests in biological reactions and physiological activities in foods are increasing recently. Peptide in food becomes a supplier of amino acid which is vital for protein biosynthesis in nutrition and has a great affect on properties of foods. Especially, serine, glycine, alanine function in reducing blood cholesterol level and have antioxidative effect and functions related to immunity (Lee et al., 1998).

Bioactive peptides can be released by the microbial activity of fermented food or through enzymes derived from microorganism (Korhonen \& Pihlanto, 2003). Therefore, fermentation is considered to be an efficient way to produce bioactive peptides and may also synthesize new peptide sequences. Numerous peptides with various bioactive functions such as antioxidative, anticancer, immunomodulatory actions, opioid agonistic and antagonistic have been identified (Chung et al., 1997; Kang et al., 2003; Mahmoud, 1994; Lee, 1998; Lee et al., 2002; Korhonen \& Pihlanto, 2003; Wenyi \& Elvira, 2005). Generally, fermentation is not enough to fully hydrolyze soybean proteins. Glycoproteins, phosphoproteins, and other modified constituents that contain a higher number of disulfide bridges are more difficult to hydrolyze, but the proteases in Bacillus and Rhizopus strains can successfully cleave soybean proteins into large peptides (Wenyi \& Elvira, 2005).

Interest in antioxidant activity of bioactive peptides generated from the fermentation of various proteins has attracted much attention in recent years.

The peptides from soybean, rice bran, oil seeds, seafood, milk and other dairy products have been extensively studied as the promising precursors of the bioactive peptides (Wenyi \& Elvira, 2005; Gibbs et al., 2004; Yoshikawa et al., 2003; Floris et al., 2003). Also, several reports have indicated that fermented chungkukjang showed stronger antimutagenic effects than nonfermented cooked soybeans (Lee et al., 2005; Chung et al., 1997; Ko et al., 1996; Kwon et al., 2002; Park et al., 2001). However, little research has been done on the antioxidant properties of chungkukjang derived peptides. Therefore, this study was to separate and characterize the antioxidant peptides from Korean fermented soybean pasta, chungkukjang.

\section{MATERIALS AND METHODS}

\section{Preparation of chungkukjang}

Soybean variety 'Taekwangkong' was grown and harvested at the field of National Institute of Crop Science, Suwon, Korea.

Chungkukjang was prepared following the traditional procedure. The 1,000 $\mathrm{g}$ of carefully selected soybeans were soaked in water overnight, and steamed for 5 hours at 12 $0^{\circ} \mathrm{C}$. After cooled to about $40^{\circ} \mathrm{C}$, bacterial inoculation of steamed soybean was conducted with $35 \mathrm{~g}$ of rice straw and incubated at $38^{\circ} \mathrm{C}$ for 48 hours in the commercial fermentation jar. Rice straw provided the fermenting microorganisms, Bacillus substilis. Fully fermented chungkukjang was freeze-dried and powdered, and then defatted with hexane by using automatic fat extraction system (Gerhardt Soxtherm 2000, German), Defatted chungkukjang powder was extracted with methanol for 24 hours, then the suspension was filtered with No 3 filter paper, and pass through by a $0.25 \mu \mathrm{m}$ PTFE filter. The filtrate was evaporated in a rotary vacuum evaporator (EYELA Co, Tokyo, Japan) prior to use.

\section{Separation of peptide fractions}

Condensed methanol extract of chungkukjang was fractionated by a recycling preparative HPLC (Japan Analytical Industry Co., Ltd., Tokyo, Japan) equipped with a Jaigel- 

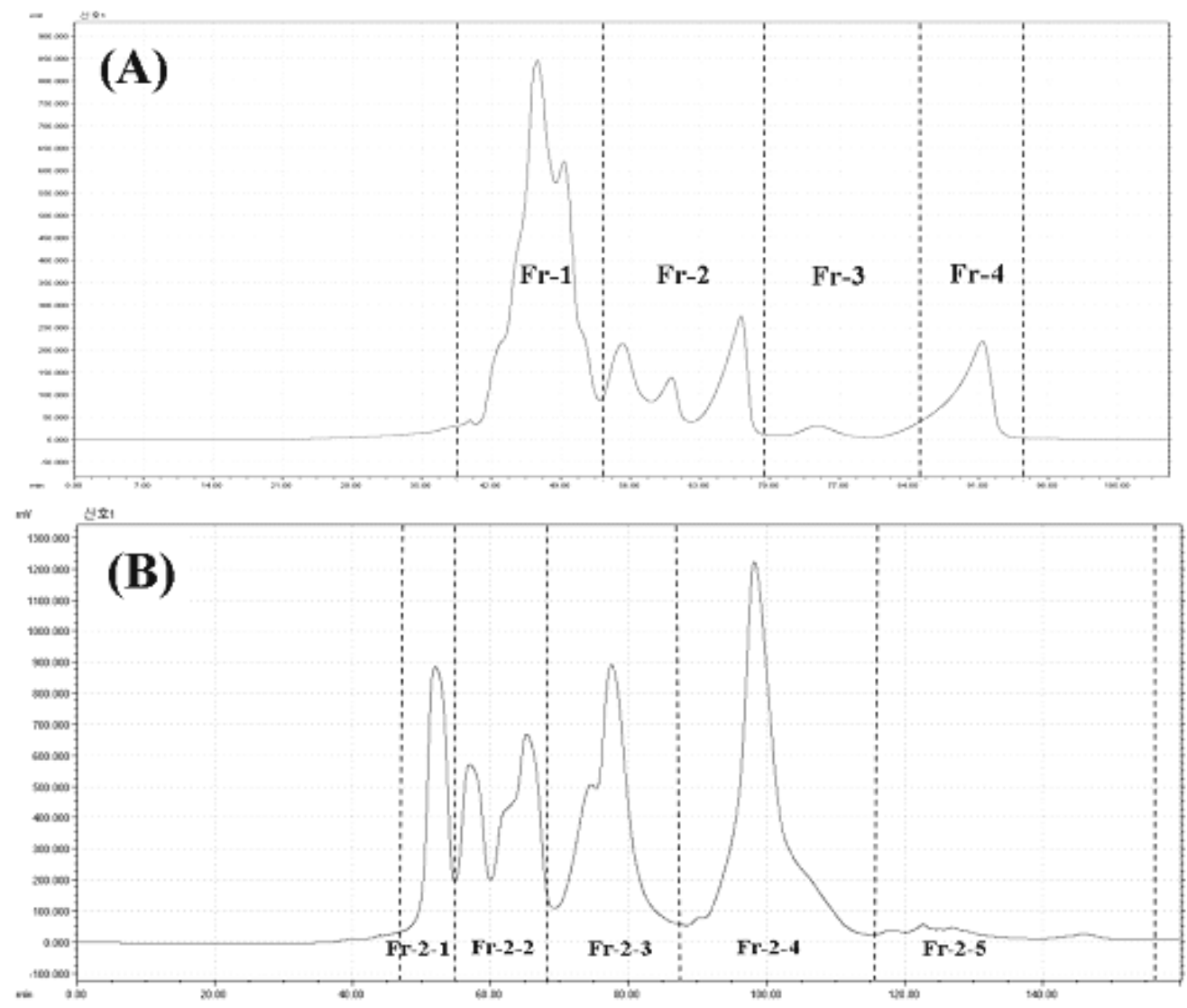

Fig. 1. Separation scheme of active fractions from methanol extract of chungkukgang by Jaigel-GS310 prep-column, and antioxidant fraction was selected by DPPH radical scavenging activity and NBT-reduction inhibition (A). Base on antioxidant effects, fraction Fr-2 was refractionated by Jaigel-W252 prep-column and five potent fractions were separated (B).

GS310 prep-column $(20 \times 500 \mathrm{~mm}$, JAI, Japan $)$.

As a mobile phase, methanol $(100 \%, \mathrm{v} / \mathrm{v})$ was applied at a flow rate of $5.0 \mathrm{ml} / \mathrm{min}$ in isocratic mode and the active fractions were detected at $280 \mathrm{~nm}$ (UV-3702, JAI, Japan). This procedure generated four fractions (Figure 1), and which were subjected to determine their antioxidant activities of DPPH free radical scavenging and NBT reduction inhibition. Considering antioxidative effects, the fractions which displayed the highest antioxidant activity were then further separated on a Jaigel-W252 prep-column $(20 \times 500$ mm, JAI, Japan). To accomplish this, methanol (100\%, $\mathrm{v} / \mathrm{v}$ ) was applied at a flow rate of $3.5 \mathrm{~mL} / \mathrm{min}$ in isocratic mode and the active fraction was collected. Finally, five potent fractions were separated (Figure 1).

\section{Assessment of radical scavenging activity}

DPPH radical scavenging activities of separated fractions were measured by 1,1-diphenyl-2-picryl-hydrazyl (DPPH). Assessment of free radical scavenging activity was conducted by measuring the absorbance of DPPH as described by Yoshida et al. (2003). The reaction mixture, which comprised $2.5 \mathrm{~mL}$ of $0.35 \mathrm{mM}$ DPPH (dissolved in $50 \%$ ethanol, $\mathrm{v} / \mathrm{v}$ ) and $0.2 \mathrm{~mL}$ of collected fractions, was incubated for 10 $\mathrm{min}$ at room temperature, and changes in DPPH absorbance were measured at $517 \mathrm{~nm}$. Absorbance of ethanol $(100 \%$, $\mathrm{v} / \mathrm{v})$ was used as a blank. The antioxidant activity was calculated as per cent inhibition caused by the hydrogen donor activity of each sample: Inhibition $(\%)=(1-a b s o r b a n c e$ of sample/absorbance of blank $) \times 100$. The NBT-reduction inhibition was measured by a spectrophotometer at $560 \mathrm{~nm}$ (Asada et al., 1974). Test tubes containing reaction solution were illuminated with 20-W Sylvania Groiux fluorescent lamps for $7 \mathrm{~min}$ at $25^{\circ} \mathrm{C}$. Antioxidant activity was represented as follows: Antioxidant activity $(\%)=(1-A / B)$ where $\mathrm{A}$ is 
the absorbance of samples and B is the absorbance of the control, respectively.

\section{Peptide characterization by reversed-phase HPLC}

Fractions were separated with a reversed-phase $C_{18}$ column $\left(4.6 \times 250 \mathrm{~mm}, 5 \mathrm{u}\right.$, Phenomenex, USA) and Millenium ${ }^{32}$ HPLC workstation system (Waters, USA). The mobile phase consisted of $0.1 \%$ trifluoroacetic acid (TFA) in water (eluent A) and of $0.1 \%$ TFA in acetonitrile (eluent B). The flow rate was $1 \mathrm{~mL} / \mathrm{min}$ and a $90 \mathrm{~min}$ gradient of $20 \sim 45 \%$ eluent B was followed by elution with $45 \%$ eluent B for $20 \mathrm{~min}$, and spectra were monitored at $210 \mathrm{~nm}$. The injection volume for all fractions was $20 \mathrm{uL}$.

\section{Amino acid analysis}

The $3 \mathrm{~mL}$ of $6 \mathrm{~N}-\mathrm{HCl}$ was added on the obtained fractions, and hydrolyzed for 24 hours at $110^{\circ} \mathrm{C}$ in test tubes with nitrogen gas flushing. Afterwards, the hydrolyzed samples were diluted to the $100 \mathrm{~mL}$ of Milli-Q water and filtered with Millipore 0.45 um syringe filters (Waters, Milford, USA). Filtrated sample was put into an autosampler and injected into an amino acid autoanalyzer (Hitachi L8800, Japan). The amount of each amino acid present in the sample was calculated with reference to the standard amino acids (Ajnomoto-Takara Co., Japan).

\section{Assessment of cell viability and DCFH-DA oxidation}

Cell viability assay was performed using the modified 3-(4,5-dimethylthiazol-2-yl) 2,5-diphenyl tetrazolium bromide (MTT) methods (Chung et al., 1997). The rat hepatoma H4IIE cell line was cultured in the medium containing $10 \%$ fetal bovine serum and 1\% penicillin/streptomycin in a humidified atmosphere with $5 \% \quad \mathrm{CO}_{2}$ at $37^{\circ} \mathrm{C}$. H4IIE cells were passaged every 3-4 days. After incubation, cells were stained with MTT $(0.2 \mathrm{mg} / \mathrm{mL})$ for 4 hours. After the medium was removed, formazan crystals formed in the wells were dissolved in $100 \mu \mathrm{L}$ dimethylsulfoxide(DMSO). The absorbance was measured at $540 \mathrm{~nm}$ using an ELISA microplate reader (Multiskan EX, Thermo Labsystems). Cell viability was calculated using the following equation: Viability $(\%$ control $)=$ absorbance of sample/(absorbance of control) $\times 100$.

For the assay of DCFH-DA oxidation, H4IIE cells $\left(1 \times 10^{6}\right.$ cells $\left./ \mathrm{mL}\right)$ were washed twice with phosphatebuffered saline and 2',7'-dichlorofluorescein diacetate (DCFHDA) was added to the cell suspension, than incubated in a $37^{\circ} \mathrm{C}$ water bath for $10 \mathrm{~min}$. After incubation, H4IIE cells were treated with peptide fractions of chungkukjang in the presence and absence of $250 \mu \mathrm{M} \mathrm{H} \mathrm{H}_{2} \mathrm{O}_{2}$-induced oxidative stress. The antioxidant activity was evaluated by measuring the intensity of dichlorofluorescein (DCF) fluorescence using fluorescence-activated cell sorting (FACS, Becton Dickinson, USA) analysis (Chi et al., 2007).

\section{RESULTS AND DISCUSSION}

\section{Separation of antioxidant peptides}

In the present study, we tried to separate the peptide fractions from the methanol extract of chungkukjang by using a recycling preparative LC.

Recycling preparative LC consists of a switching-column which is accomplished by permitting the eluent to be directed from the column outlet back into the column inlet. Therefore, the separation process among peaks can be repeated to increase the effective separation power. For this, recycling preparative LC has been successfully applied to separate natural products and compounds (Martin et al., 1976; Grill, 1998), but little has been done on the separation of peptides in the fermented soybean foods. Methanol extract of chungkukjang was loaded on Jaigel-GS310 prep-column and fractionated, and this work repeated until the peaks separated.

The columns applied to this study are a kind of gel filtration chromatography (GFC) column. They are based on the discrimination of individual components by the pores of the packing material. Therefore, large molecules can only partially penetrate the pores, whereas smaller molecules can access most of all pores. Thus, large molecules elute first, smaller molecules elute later.

As shown in Figure 1A, four fractions were collected with GS310 column, and they were subjected to determine the antioxidant activity according to the methods of DPPH free radical scavenging activity and NBT-reduction inhibition.

Among four fractions, the second fraction Fr-2 was identified to be highly potent in the assay of DPPH free radical scavenging activity and NBT-reduction inhibition. 
Base on antioxidative effects, fraction Fr-2 was employed for the refraction with a Jaigel-W252 prep-column which has been frequently used for the removal of aggregates in a final polishing step. This procedure generated five fractions (Figure 1B), and they were also subjected to antioxidant activity assay according to the same methods. Considering the antioxidant activity assay results, two antioxidant peptide fractions were finally selected, then their amino acid constituents and molecular properties were determined by using amino acid autoanalyzer and reversed-phase HPLC, respectively.

\section{Radical scavenging activity of separated fractions}

The fractions separated from the methanol extract of chungkukjang by using GS310 and W252 prep-column were subjected to assay the antioxidant activities.

$\mathrm{DPPH}$ is a relatively stable organic radical that shows maximum absorbance at $517 \mathrm{~nm}$, thus widely used as a substrate to evaluate the efficacy of antioxidants (Shimada et al., 1992; Nanjo et al., 1996). In our DPPH assay, separated fractions from the methanol extract of chungkukjang reduced the DPPH radical to a yellow-colored compound, apparently due to the DPPH radical accepting an electron or a hydrogen to become a stable diamagnetic molecule. The antioxidant activity of the second fraction Fr-2 was significantly higher as compared with other separated fractions in two methods. Fraction Fr-2 showed approximately $72.4 \%$ of radical scavenging activity in DPPH, and $81.4 \%$ in NBT reduction inhibition assay (Figure 2).

According to the antioxidant activity assay results, fraction Fr-2 was selected for the refractionation on a W252 prep-column, then finally separated into five fractions, and these partially purified peptide fractions were also subjected to antioxidant activity assay.

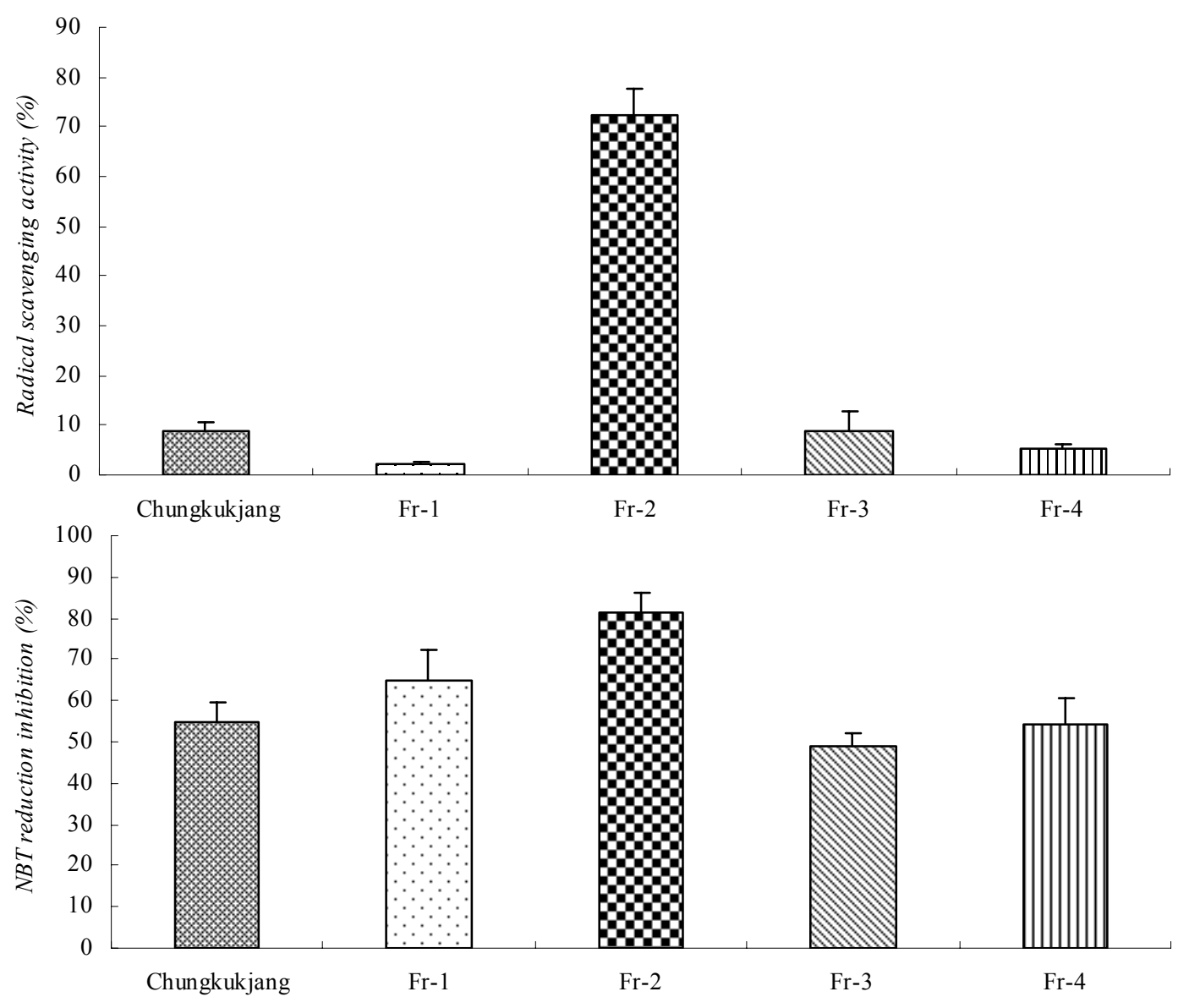

Fig. 2. DPPH radical scavenging activity (upper panel) and NBT-reduction inhibition (lower panel) of separated fractions from methanol extract of chungkukjang. 

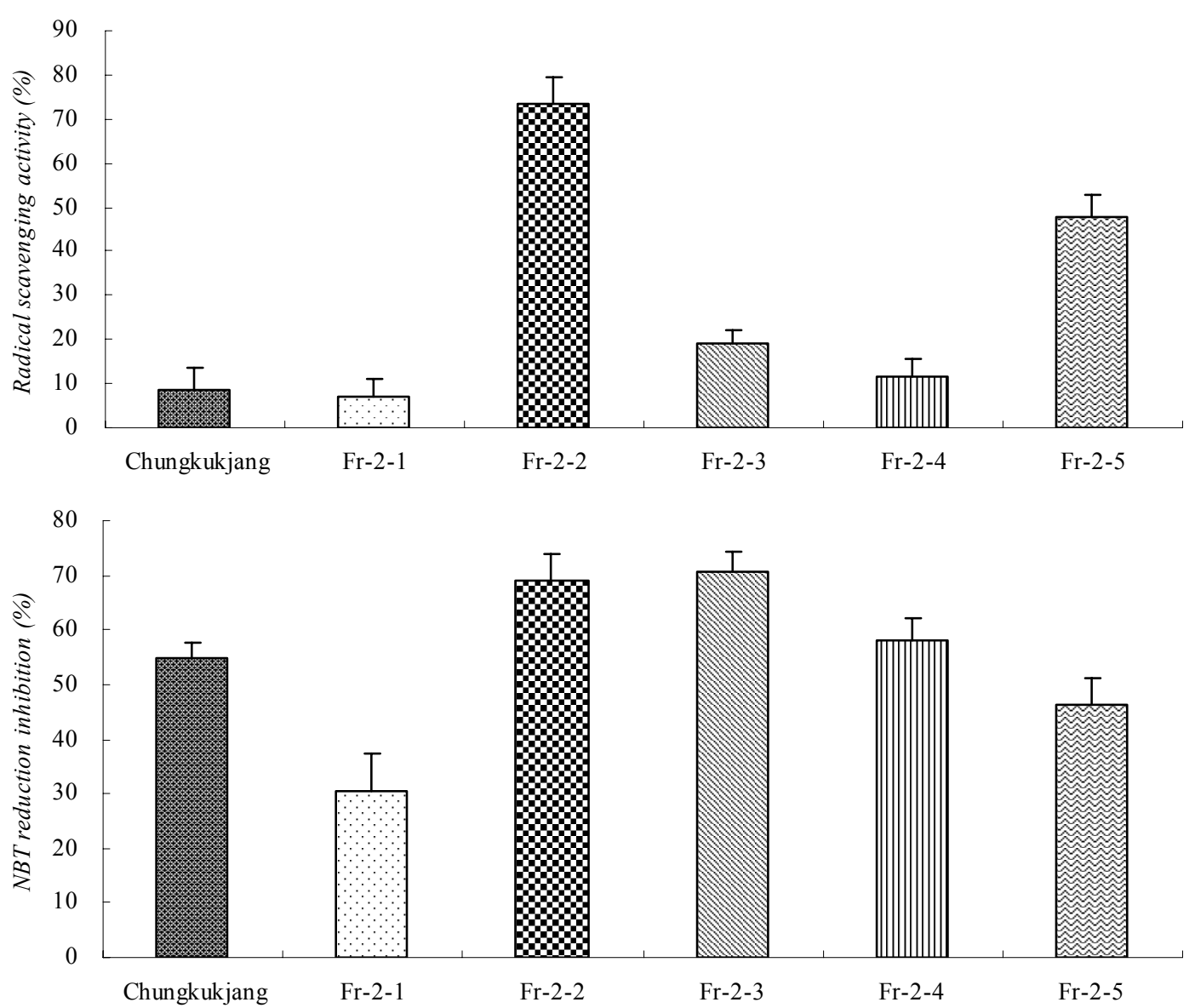

Fig. 3. DPPH radical scavenging activity (upper panel) and NBT-reduction inhibition (lower panel) of separated peptide fractions, Fr-2-2 and Fr-2-3.

Among the five fractions, fraction Fr-2-2 was observed to be the most potent antioxidant activity of $73.3 \%$ in DPPH assay, furthermore fractions Fr-2-2 and Fr-2-3 showed their activities $69.1 \%$ and $70.5 \%$ in NBT reduction inhibition assay, respectively (Figure 3).

Several peptides are generally accepted to be antioxidative activity. Chen et al. (1998) reported soybean peptides have higher antioxidant activity than intact soybeans. Matoba (2002) also reported that after enzyme digestion of $\beta$ conglycinin and glycinin, the radical-scavenging activities were increased 3 to 5 times than intact soybean protein.

Fermentation is considered to be an efficient way to produce bioactive peptides. Bioactive peptides are released by the microbial activity of fermented food or through enzymes derived from microorganism (Korhonen \& Pihlanto, 2003).

Therefore, this study results suggest that the peptide fractions separated from the methanol extract of chungkukjang have an antioxidant activity.

\section{Peptide characterization by reversed-phase HPLC}

We examined the peptide properties of selected antioxidant fractions by using analytical HPLC, and obtained results were presented in Figure 4.

The column utilized in the investigation was reversedphase $\mathrm{C}_{18}(4.6 \times 250 \mathrm{~mm}, 5 \mathrm{u})$ column designed for separation of proteins and peptides (> 10,000 MW).

As shown in Figure 4, the fractions were composed of various peaks, but the HPLC elution profiles of two fractions Fr-2-2 and Fr-2-3 were apparently different between each other. Generally, peptide chromatographic profiles are determined by the characters of their side chains and substituent groups, which define their basic or acidic character, or the degree of hydrophobicity or hydrophilicity 

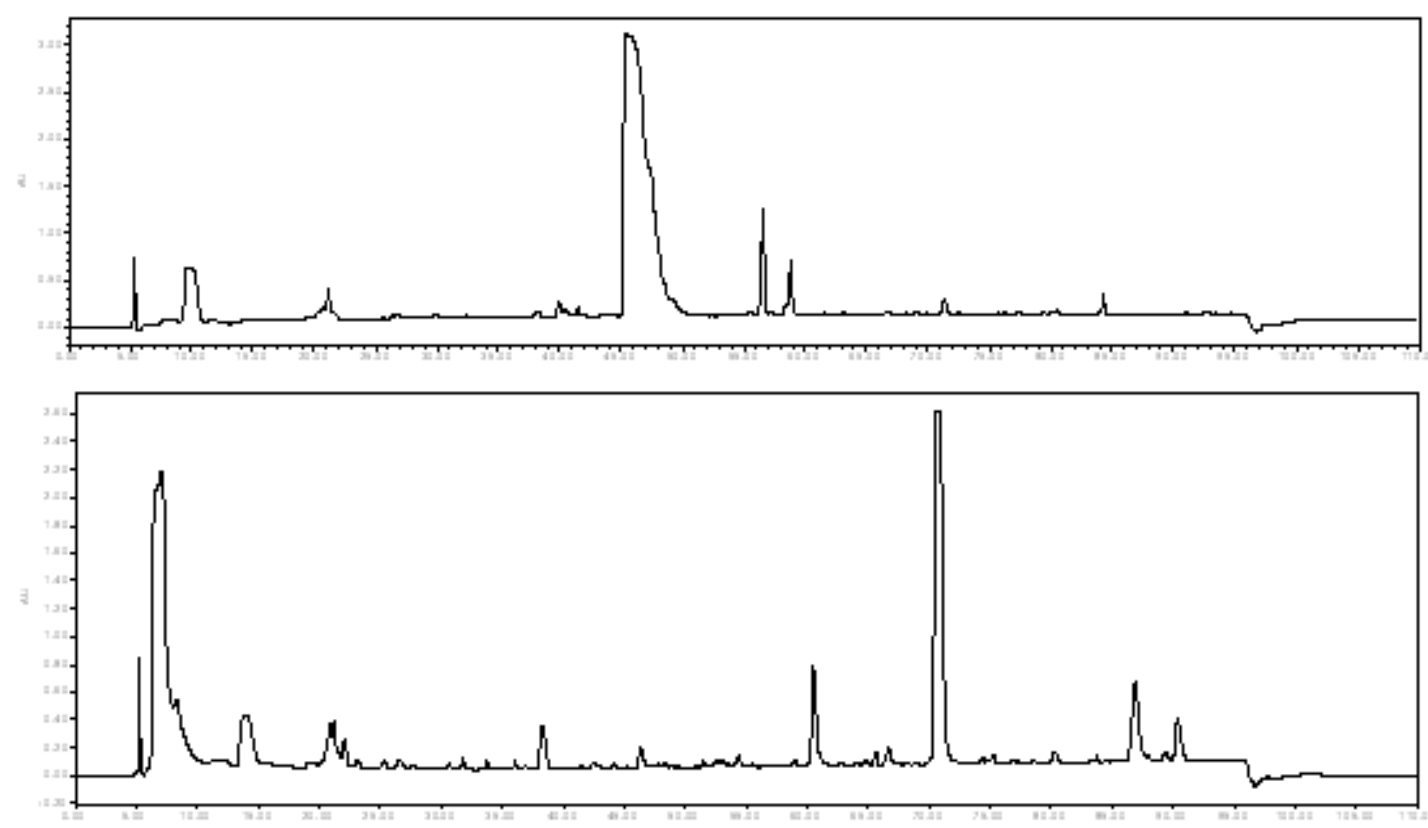

Fig. 4. Reversed-phase HPLC chromatogram profiles on a Jupiter C18 column $(4.6 \times 250 \mathrm{~mm}, 5 \mu)$ of antioxidant fractions Fr-2-2 (upper chromatogram) and Fr-2-3 (lower chromatogram) which were fractionated by a recycling preparative LC equipped with a Jaigel-W252 prep-column $(20 \times 500 \mathrm{~mm})$ as shown in Figure 1 . Chromatogram was obtained by using a $0.1 \%$ TFA in water (eluent A) and $0.1 \%$ TFA in acetonitrile (eluent B). Flow rate was $1 \mathrm{~mL} / \mathrm{min}$ and a 90 min gradient of $20 \sim 45 \%$ eluent B was followed by elution with $45 \%$ eluent B for $20 \mathrm{~min}$, and spectra were monitored at $210 \mathrm{~nm}$. The injection volume was $20 \mathrm{uL}$.

(Bogusław et al., 2007). Moreover, the retention times of peptides are depended on specific interactions between stationary phase, mobile phase, and the investigated compounds. Therefore, as observed in the present study, different elution profiles of antioxidant fractions on retention can be explained the properties of peptide fractions. As presented in Figure 1, two antioxidant fractions Fr-2-2 and Fr-2-3 were separated with Jaigel-W252 prep-column, thus the large peptide molecules had eluted first, while the smaller peptide molecules had eluted later.

However, the reversed-phase HPLC elution profiles of two fractions were completely different from the profiles of a Jaigel-W252 prep-column. In general, the smaller and hydrophilic molecules were eluted earlier on the reversedphase $\mathrm{C}_{18}$ column. Therefore, this study results implied that the antioxidant peptide fraction Fr-2-2 is composed of relatively large molecules and more potent hydrophobicity than those of fraction Fr-2-3. However, further investigations are required to clarify the nature of the peptide fractions.

\section{Amino acid composition of chungkukjang and their antioxidant fractions}

The amino acid compositions of chungkukjang, and their antioxidant fractions Fr-2-2 and Fr-2-3 were analyzed by amino acid autoanalyzer.

Eighteen amino acids were identified in chungkukjang (Figure 5). The obtained results showed that acidic amino acids such as glutamic and aspartic acid constitute approximately $32.6 \%$ of the total amino acids, and glutamic acid is the most abundant amino acid in chungkukjang. It is generally accepted that the specific savory taste of chungkukjang mainly comes from glycine, glutamic acid, arginine, and other free amino acids. Lee (1973) and An et al. (1987) also reported that fermented soybean products had more glutamic acid and aspartic acid in amino acid than soybeans, therefore our study results agreed with these findings.

To confirm the amino acid constituents of antioxidant fractions Fr-2-2 and Fr-2-3 were analyzed, and Figure 6 presented the results. 


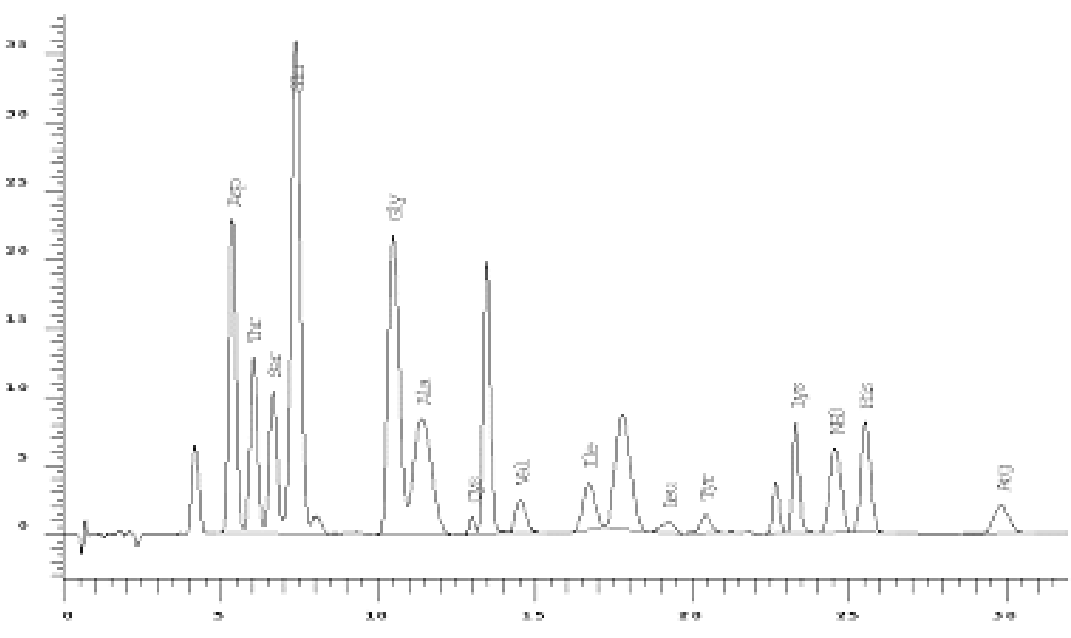

\begin{tabular}{lc}
\hline \hline Amino acids & Composition (\%) \\
\hline Aspartic acid & $12.68 \pm 0.71$ \\
Threonine & $6.13 \pm 1.24$ \\
Serin & $5.73 \pm 0.36$ \\
Glutamic acid & $19.93 \pm 1.19$ \\
Glycine & $10.14 \pm 0.80$ \\
Alanine & $4.70 \pm 0.45$ \\
Cystein & $0.70 \pm 0.22$ \\
Valine & $9.01 \pm 1.26$ \\
Methionine & $1.31 \pm 0.09$ \\
Isoleucine & $1.87 \pm 0.24$ \\
Leucine & $4.65 \pm 0.62$ \\
Tyrosine & $0.38 \pm 0.06$ \\
Phenylalanine & $2.79 \pm 1.63$ \\
Lysine & $4.46 \pm 0.08$ \\
Histidine & $4.47 \pm 0.55$ \\
Arginine & $4.15 \pm 0.42$ \\
Proline & $6.90 \pm 0.75$ \\
\hline
\end{tabular}

Fig. 5. Amino acid composition of Korean fermented soybean paste, chungkukjang.

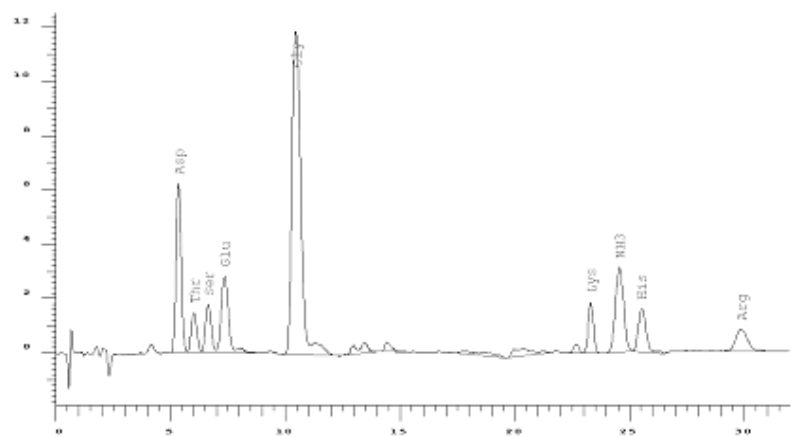

\begin{tabular}{lrr}
\hline \hline \multirow{2}{*}{ Amino acids } & \multicolumn{2}{c}{ Composition (\%) } \\
\cline { 2 - 3 } & \multicolumn{1}{c}{ Fr-2-2 } & \multicolumn{1}{c}{ Fr-2-3 } \\
\hline Aspartic acid & $15.91 \pm 0.16$ & $14.12 \pm 0.13$ \\
Threonine & $4.11 \pm 0.40$ & $6.61 \pm 0.46$ \\
Serine & $5.13 \pm 0.09$ & $7.43 \pm 0.05$ \\
Glutamic acid & $9.70 \pm 0.52$ & $15.04 \pm 0.39$ \\
Glycine & $49.82 \pm 3.45$ & $36.60 \pm 2.87$ \\
Lysine & $4.68 \pm 0.11$ & $7.20 \pm 0.55$ \\
Histidine & $6.02 \pm 0.21$ & $7.91 \pm 0.65$ \\
Arginine & $4.62 \pm 0.31$ & $5.09 \pm 0.25$ \\
\hline
\end{tabular}

Fig. 6. Amino acid compositions of antioxidant peptide fractions, Fr-2-2 (upper chromatogram) and Fr-2-3 (lower chromatogram) which were separated from Korean fermented soybean paste, chungkukjang.

Eight kinds of amino acids such as aspartic acid, threonine, serine, glutamic acid, glycine, lysine, histidine, and arginine were identified as the constituent amino acids of two peptide fractions. Among the eight amino acids, glycine was revealed as the most abundant constituent amino acid, and considerable amounts of aspartic acid, glutamic acid, histidine, serine, lysine, arginine, and threonine were found in Fr-2-2 and Fr-2-3, respectively.

Several peptides are generally accepted have an antioxidative activity, and these peptides usually composed of 2-20 amino acid residues per molecule, and the lower the molecular weight, the higher their chance to exert biological effects (Pihlanto-Leppala, 2001; Roberts et al., 1999). The peptides derived from soybean protein hydrolysate 
with 5-16 amino acid residues showed strong inhibition activities on the autoxidation of linoleic acid (Chen et al., 1995). Kim et al. (1999) have obtained a glycopeptide from ethanol fractions of bromelain-defatted soybeans hydrolysate composed mainly of Asp, Glu, Pro, Gly and Leu with strong cytotoxic activity against P388D1 mouse lymphoma cells. Hydrophobic amino acids and one or more residues of His, Pro, Met, Cys, Tyr, Trp, Phe and Met are believed to enhance the activities of the antioxidant peptides (Chen et al., 1998; Da'valos et al., 2004; Herna'ndez-Ledesma et al., 2005). Among the listed amino acids, histidine, methionine and cysteine are very important to the radical scavenging activity of peptides due to their special structure of characteristics. Histidine has the proton-donation ability, while methionine is prone to oxidation of the methionine sulfoxide, and cysteine donates the sulfur hydrogen (Herna'ndezLedesma et al., 2005; Tsuge et al., 1991). Some recent reports suggested that the peptide Gln-Gly-Ala-Arg, which exhibited the highest antioxidant activity from porcine skin collagen hydrolysates (Li et al., 2007; Suetsuna et al., 2000), and the Glu-Leu residue in peptides was recently reported to play an important role in radical scavenging (Jun et al., 2004). Additionally, presence of aspartic acid seems to play a vital role irrespective of its position as observed in several antioxidative peptide sequences (Byuna et al., 2009).
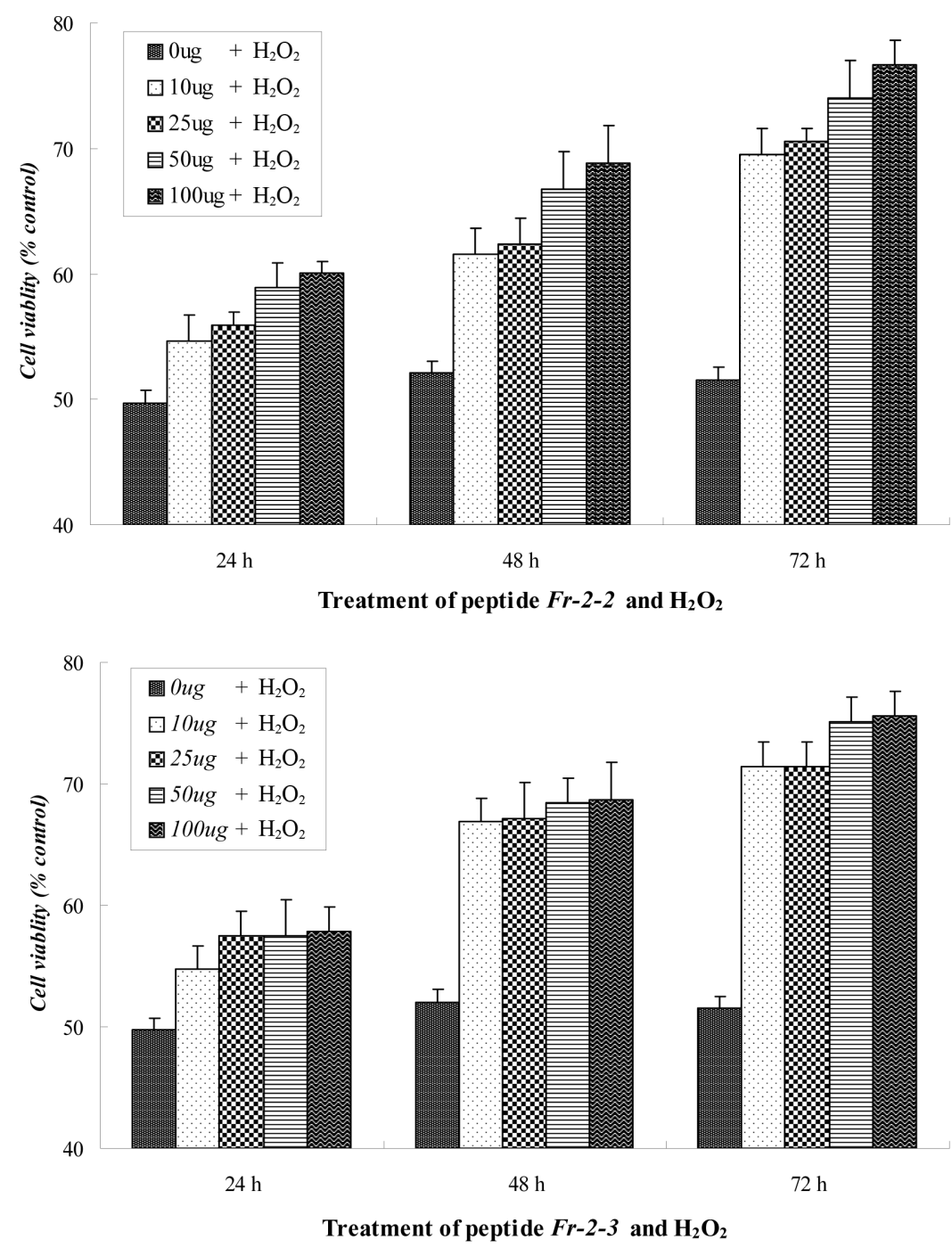

Fig. 7. Concentration and time-dependent antioxidant effects on H4IIE cells which were treated with $\mathrm{H}_{2} \mathrm{O}_{2}$-induced oxidative stress. Cell viability was expressed relative to the untreated control cell. Each value represents the mean $\pm \mathrm{SD}$. 
In the present study, we identified the eight amino acids (Gly, Asp, Glu, His, Ser, Lys, Arg, and Thr). Therefore, we speculate that these amino acids might play an important role on their antioxidant activity of the two peptide fractions Fr-2-2 and Fr-2-3.

The antioxidant activity of peptides is closely related to their amino acid constituents and their sequences (Chen et al., 1998; Suetsuna et al., 2000). However, this study didn't specifically investigate the amino acid sequence due to the separated fractions were overlayed with other peaks (Figure 1). Therefore, to obtain more information about antioxidant peptides derived from chungkukjang, further investigations of the amino acid sequences of the active peptide fractions Fr-2-2 and Fr-2-3 were required.

\section{Effect of chungkukjang peptides on cell viability and DCFH-DA oxidation}

In order to examine the effect of peptide fractions to protect from cell death, a MTT assay was performed using rat hepatoma H4IIE cell line.

Base on a preliminary MTT assay result (data not shown), we determined an optimal incubation condition that maintains approximately $50 \%$ cell viability upon exposure of the cells to $1 \mathrm{mM} \mathrm{H}_{2} \mathrm{O}_{2}$ stress.

In the absence of peptide fractions, cell viability was approximately $50 \%$ after the treatment of $\mathrm{H}_{2} \mathrm{O}_{2}$-induced oxidative stress. However, in the presence of peptide fractions, cell viability was remarkably higher as increasing the concentrations of peptides from 10 to $100 \mu \mathrm{g} / \mathrm{mL}$, although the H4IIE cells were exposed to the $\mathrm{H}_{2} \mathrm{O}_{2}$-induced oxidative stress (Figure 7).

These results strongly suggested that the peptide fractions Fr-2-2 and Fr-2-3 have a significant effect on the cell viability in a concentration and time dependent manner. Therefore, the ability of chungkukjang peptides to protect from cell death was considered mainly due to their potent antioxidant activity that reduces the oxidative stress.

Conversion of non-fluorescent dichlorofluorescein diacetate
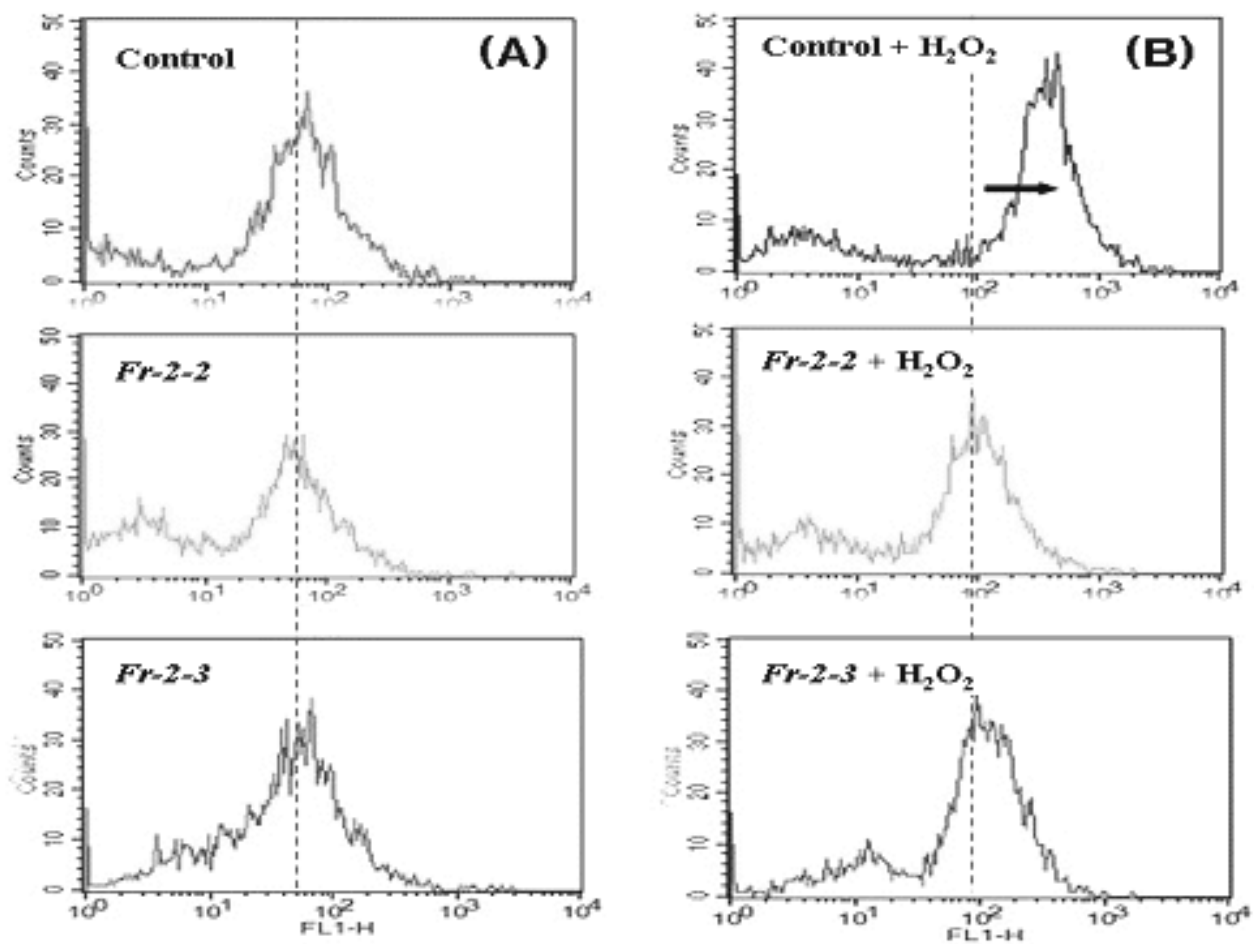

Fig. 8. Fluorescence activated cell sorter (FACS) analysis of chungkukjang peptide fractions. H4IIE cells were treated with peptide fractions in the presence and absence of $250 \mu \mathrm{M} \mathrm{H}_{2} \mathrm{O}_{2}$-induced oxidative stress. (A): H4IIE cells treated with peptide fractions, and control, $\mathrm{Fr}$-2-2 and Fr-2-3. (B): H4IIE cells treated with peptide fractions and $\mathrm{H}_{2} \mathrm{O}_{2}$-induced oxidative stress. 
(DCFH-DA) to fluorescent dichlorofluorescein (DCF) by hydrogen peroxide can be detected by a fluorescence activated cell sorter (FACS) assay. Once DCFH-DA is taken up by living cells, it is deacetylated to nonfluorescent dichlorofluorescein by the action of esterase. And, subsequent oxidation of DCFH by ROS produces DCF which can be easily visualized (Halliwell \& Gutteridge, 1999). As shown in Figure $8 \mathrm{~A}, \mathrm{H}_{2} \mathrm{O}_{2}$-treated H4IIE cells increased DCF fluorescence significantly as compared with the control H4IIE cells.

However, in the addition of fractions Fr-2-2 and Fr-2-3, the DCF fluorescence of H4IIE cells was not increased, although the cells were exposed to $\mathrm{H}_{2} \mathrm{O}_{2}$-induced oxidative stress (Figure 8B). These results were well consistent with the results obtained by the observation of the antioxidant activity assays of DPPH scavenging activity, NBTreduction inhibition, and MTT cell viability.

Therefore, the presented study results indicated that fermented soybean paste chungkukjang will promote the antioxidant and radical scavenging activities, and beneficial for health. The antioxidant peptide fractions Fr-2-2 and Fr-2-3 were denominated as P-NICS-1 and P-NICS-2, respectively, and further studies are under way in our laboratory to clarify the amino acid sequences and molecular properties, and physiological significances of these peptides.

\section{REFERENCES}

An, H. S., J. S. Bae, and T. S. Lee. 1987. Comparison of free amino acids, sugars, and organic aicd in soybean paste prepared with various organisms. J. Korean Agricultural Chemical. 30 : 345-350.

Asada, K., M. Takahashi, and M. Nagate. 1974. Assay and inhibitors of spinach superoxide dismutase. Agr. Biol. Chem. 38 : 471-173.

Bogusław, B., K. Sylwia, K. Tomasz, R. Katarzyna, M. Monika, and J. Tobias. 2007. HPLC columns partition by chemometric methods based on peptides retention. $J$. Chromatogr. B. 845 : 253-260.

Byuna, H. G., L. K. Lee, H. G. Park, J. K. Jeon, and S.K. Kim. 2009. Antioxidant peptides isolated from the marine rotifer, Brachionus rotundiformis. Process Biochemistry. 44 : 842-846.

Chen, H. M., K. Muramoto, and F. Yamaguchi. 1995. Structural analysis of antioxidative peptides from soybean b-conglycinin. J. Agric. Food Chem. 43 : 574-578.
Chen, H. M., K. Muramoto, F. Yamaguchi, K. Fujimoto, and K. Nokihara. 1998. Antioxidative properties of histidinecontaining peptides designed from peptide fragments found in the digests of a soybean protein. J. Agric. Food Chem. $46: 49-53$.

Chi, H. Y., C. H. Lee, K. H. Kim, S. L. Kim, and I. M. Chung. 2007. Analysis of phenolic compounds and antioxidant activity with H4IIE cells of three different rice grain varieties. Eur. Food Res. Technol. 225 : 887-893.

Choe, J. S., J. S. Kim, S. M. Yoo, H. J. Park, T. Y. Kim, C. M. Chang, and S. Y. Shin. 1996. Survey on preparation method and consumer response of Chung-Kuk-Jang. Korea Soybean. Digest. 13 : 29-43.

Chung, K. S., K. D. Yoon, D. J. Kwon, S. S. Hong, and S. Y. Choi. 1997. Cytotoxicity testing of fermented soybean products with various tumour cells using MTT assay. Kor. J. Appl. Microbiol. Biotechnol. 28 : 239-241.

Da'valos, A., M. Miguel, B. Bartolome', and R. Lo'pezFandino. 2004. Antioxidant activity of peptides derived from egg white proteins by enzymatic hydrolysis. J. Food Protection. 67 : 1939-944.

Floris, R.; I. Recio, B. Berkhout, and S. Visser. 2003. Antibacterial and antiviral effects of milk proteins and derivatives thereof. Curr. Pharm. Des. 9 : 1257-1275.

Fujii, H., A. Shiraishi, H. Kaba, M. Shibagaki, S. Takahashi, and A. Honda. 1975. In topics in secondary metabolism I Bacillus subtillis: Molecular biology and industrial application. Hakkokogaku Kaishi. 53 : 424.

Gibbs, B. F., A. Zougman, R. Masse, and C. Mulligan. 2004. Production and characterization of bioactive peptides from soy hydrolysate and soy-fermented foods. Food Res. Int. 37 : 123-131.

Grill, C. M. 1998. Closed-loop recycling with periodic intraprofile injection: a new binary preparative chromatographic technique. J. Chromatogr. $796: 101-113$.

Halliwell, B., J. and M. C. Gutteridge. 1999. Free radicals in biology and medicine, 3rd ed. Oxford University Press, Oxford. p. 381-385.

Herna'ndez-Ledesma, B., A. Da'valos, B. Bartolome', and L. Amigo. 2005. Preparation of antioxidant enzymatic hydrolysates from clactalbumin and a-lactoglobulin, identification of active peptides by HPLC-.MS/MS. J. Agric. Food Chem. 53 : 588-593.

Jun, S. Y., P. J. Park, W. K. Jung, and S. K. Kim. 2004. Purification and characterization of an antioxidative peptide from enzymatic hydrolysate of yellowfin sole (Limanda aspera) frame protein. Eur. Food Res. Technol. 219 : 20-26.

Kang, M. J., J. I. Kim, and T. W. Kwon. 2003. Effect of chongkukjang on blood glucose and lipid profile in neonatal streptozotocin-induced diabetic rats. Food Sci. Biotechnol. 12 : 544-547.

Kim, J. Y., H. J. Woo, C. W. Ahn, H. S. Nam, Z. I. Shin, 
and H. J. Lee. 1999. Cytotoxic effects of piptides fractionated from bromelain hydrolyzates of soybean protein. Foo Sci. Biotechnol. 8 : 333-337.

Kim, W., K. Choi, Y. Kim, H. Park, J. Choi, Y. Lee, H. Oh, I. Kwon, and S. Lee. 1996. Purification and characerterization of fibrinolytic enzyme produced form Bacillus sp strain CK-11-4 screened from chungkukjang. Appl. Environ. Microbiol. 62 : 2482-2488.

Ko, H. S.; H. K. Ju, K. O. Jung, and K. Y. Park. 1996. Antimutagenic effect of chungkookjangs prepared with the different kinds of soybeans. J. Korean Assoc. Cancer Preven. 4 : 204-212.

Korhonen, H., and A. Pihlanto. 2003. Food-derived bioactive peptides-opportunities for designing future foods. Curr. Pham. Des. 9 : 1297-1308.

Kwon, E. Y., K. O. Jung, S. H. Moon, and K. Y. Park. 2002. Studies on enhancing chemopreventive effect of chungkookjangsAntimutagenic activity of chungkookjangs prepared with the different fermentation periods and ingredient ratio. $J$. Korean Assoc. Cancer Preven. 7 : 200-209.

Lee, B. Y., D. M. Kim, and K. H. Kim. 1991. Physicochmical properties of viscous substances extracted from chungkookjang. Korean J. Food Sci. Technol. 23 : 599-604.

Lee, C. H. 1973. Studies on the amion acid composition of Korean fermented soybean Meju products and the evaluation of the protein qualiy. Korean J. Food Sci. Technol. 5 : 210-214.

Lee, H. J. 1998. Health fuctional peptides form soyben foods. Korea Soybean Digest. 15 : 16-22.

Lee, M. Y., S. Y. Park, K. O. Jung, K. Y. Park, and S. D. Kim. 2005. Quality and functional characteristics of chungkukjang prepared with various Bacillus sp. isolated from traditional chungkukjang. J. of Food Sci. 70 : 191-196.

Lee, S. H., B. N. Cho, C. K. Hyun, and S. S. Jew. 2002. Physiological, functional characterisetics of silk peptide: Antioxidant effect and immune fuction. Food Science and Industry. 35 : 57-62.

Lee, S. K.; S. Heo, D. H. Bae, and K. H. Choi. 1998. Medium optimization for fibrinolytic enzyme production by Bacillus subtilis KCK-7 isolated from Korean traditional chungkukjang. J. Appl. Microbiol. Biotechnol. 26 : 226-231.

Li, B., F. Chen, X. Wang, B. Ji, and Y. Wu. 2007. Isolation and identification of antioxidative peptides from porcine collagen hydrolysate by consecutive chromatography and electrospray ionization mass spectrometry. Food Chem. 102 : 1135-1143.

Mahmoud, M. I. 1994. Physicochemical and fucctional properties of protein hydrolysates in nutritional products. Food Technology. 48 : 89-95.

Martin, M., F. Verillon, C. Eon, and G. Guiochon. 1976.
Theoretical and experimental study of recycling in high performance liquid chromatography. J. Chromatogr. 125 : 17-41.

Matoba, T. 2002. How does the radical-scavenging activity of soy protein food change during heating. Daizu Tanpakushitsu Kenkyu. 5 : 47-50.

Nanjo, F., K. Goto, R. Seto, M. Suzuki, M. Sakai, and Y. Hara. 1996. Scavenging effects of tea catechins and their derivatives on 1,1,-diphenyl-2-picrylydrazyl radical. Free Radical Biology and Medicine. 21 : 895-902.

Park, K. Y., E. Y. Kwon, and K. O. Jung. 2001. Studies on enhancing chemopreventive effect of chungkookjangs. Antimutagenic activity of chungkookjangs prepared with the different varieties of soybean and starter. J. Korean Assoc Cancer Preven. 6 : 36-43.

Pihlanto-Leppala A. 2001. Bioactive peptides derived from bovine whey proteins: opioid and ACE-inhibitory peptides. Trends Food Sci. Tech. 11 : 347-356.

Roberts, P. R., J. D. Burney, K. W. Black, and G. P. Zaloga. 1999. Effect of chain length on absorption of biologically active peptides from the gastrointestinal tract. Digestion. 60 : $332-337$.

Shimada, K., K. Fujikawa, K. Yahara, and T. Nakamura. 1992. Antioxidative properties of xanthan on the antioxidation of soybean oil in cyclodextrin emulsion. J. Agric. Food Chem. $40: 945-948$.

Suetsuna, K., H. Ukeda, and H. Ochi. 2000. Isolation and characterization of free radical scavenging activity peptides derived from casein. J. Nutrition and Biochemistry. 11 : 128-131.

Thomas, C., M. Michèle, and F. Agnès. 2005 CapE, a 47-amino-acid peptide, is necessary for Bacillus anthracis polyglutamate capsule synthesis. J. of Bacteriology. 187 : 7765-7772.

Tsuge, N., Y. Eikawa, Y. Nomura, M. Yamamoto, and K. Sugisawa. 1991. Antioxidant activity of peptides prepared by enzymatic hydrolysis of egg-white albumin. Nippon Nogeikagaku Kaishi. 65 : 1635-1641.

Wenyi, W., and G. M. Elvira. 2005. A new frontier in soy bioactive peptides that may prevent age-related chronic diseases. CRFSFS. 4 : 63-78.

Yoshida, T., K. Mori, T. Hatano, T. Okumura, I. Uehara, K. Komagoe, Y. Fujita, and T. Okuda. 1989. Studies on inhibiton mechanism of autooxidation by tannins and related polyphenols on 1.1-diphenyl-2-picrylhydrazyl radical. Chem. Pharm. Bull. 37 : 1919-1923.

Yoshikawa, M., M. Takahashi, and S. Yang. 2003. Delta opioid peptides derived from plant proteins. Curr. Pharm. Des. 9 : 1325-1330. 